\title{
Deep learning based approach for optic disc and optic cup semantic segmentation for glaucoma analysis in retinal fundus images
}

Original Scientific Paper

\author{
Dunja Božić-Štulić \\ University of Split, \\ Faculty of Electrical Engineering, Mechanical Engineering and Naval Architecture \\ Ruđera Boškovića 32, Split, Croatia \\ dgotovac@fesb.hr
}

\author{
Maja Braović \\ University of Split, \\ Faculty of Electrical Engineering, Mechanical Engineering and Naval Architecture \\ Ruđera Boškovića 32, Split, Croatia \\ mbraovic@fesb.hr

\section{Darko Stipaničev} \\ University of Split, \\ Faculty of Electrical Engineering, Mechanical Engineering and Naval Architecture \\ Ruđera Boškovića 32, Split, Croatia \\ dstip@fesb.hr
}

\begin{abstract}
Optic disc and optic cup are one of the most recognized retinal landmarks, and there are numerous methods for their automatic detection. Segmented optic disc and optic cup are useful in providing the contextual information about the retinal image that can aid in the detection of other retinal features, but it is also useful in the automatic detection and monitoring of glaucoma. This paper proposes a deep learning based approach for the automatic optic disc and optic cup semantic segmentation, but also the new model for possible glaucoma detection. The proposed method was trained on DRIVE and DIARETDB1 image datasets and evaluated on MESSIDOR dataset, where it achieved the average accuracy of $97.3 \%$ of optic disc and $88.1 \%$ of optic cup. Detection rate of glaucoma diesis is $96.75 \%$.
\end{abstract}

Keywords - optic disc, optic cup, glaucoma, deep learning

\section{INTRODUCTION}

Optic disc and optic cup are one of the most recognized retinal landmarks, alongside fovea, macula and retinal blood vessels. It is a point of convergence for retinal blood vessels, and in a fundus image that shows no abnormalities is usually represented by the brightest region in the image, A large number of methods for its automatic detection is reported in the literature (e.g. [1-5]), but approaches based on deep learning, such as the one proposed in paper, are still relatively novel. It can be used in the automatic segmentation of retinal blood vessels, fovea and macula, as it can provide contextual information about the retinal image. It is also useful in the automatic detection and monitoring of glaucoma, and eye disease usually characterized by an increased intraocular pressure that can lead to vision loss if left untreated. Medical tests for glaucoma should, amongst other things, include measurement of change in the cup-to-disc (CDR) ratio, and this ratio can be calculated automatically (e.g. [6, 7]) given the segmented optic cup and optic disc from retinal fundus image. The focus of this paper is on the automatic optic disc segmentation and optic cup segmentation. Our method is based on popular SegNet [8] deep neural network, which is originally used for semantic segmentation of natural landscape images. SegNet belongs to encoderdecoder type of deep architecture for multi-class pixelwise segmentation.

This paper is organized as follows. In Section 2. we discuss related work, with special attention being given to optic disc segmentation methods that are based on 
deep learning. In Section 3. we give an overview of the proposed method, including data preparation process and network architecture description. In Section 4. we present and discuss optic disc and cup segmentation results obtained with the proposed method, as a glaucoma detection rate. In Section 5. we give a conclusion and discuss future work.

\section{RELATED WORK}

In this section of the paper we discuss existing methods for optic disc segmentation and optic cup segmentation.

\subsection{OPTIC DISC SEGMENTATION}

Approaches for automatic optic disc segmentation span a great number of categories, and since these categories are usually intertwined, it makes it difficult to categorize these approaches into fixed categories. For this reason, in this section of the paper we discuss various techniques that are commonly used in automatic optic disc segmentation. Special attention is given to novel automatic optic disc segmentation approaches that are based on deep learning.

Automatic optic disc segmentation methods usually consist of a preprocessing part and the main segmentation part. Preprocessing part commonly encompasses the selection of the appropriate color space or color channel in which to perform further analysis, removal of retinal blood vessels from fundus images, and various techniques for image enhancement. The main segmentation part commonly consists of a combination of various image processing techniques, for example mathematical morphology, edge detection, different classifiers, etc.

One of the usual first steps in the preprocessing part of automatic optic disc detection methods is the selection of optimal color space or color channel to use. We found that various approaches use different color channels. For example, green channel from the RGB color space was used in $[9,10]$, both green and red channels from the RGB color space were used in [11] and in [12] it was found that it was reliable to use luminance channel from the HLS color space for optic disc localization and red channel from the RGB color space to find the contours of the optic disc. From this we can conclude that there is no formal agreement between researchers on what color space or color channel to use in automatic optic disc segmentation.

Another important step that is often used in the preprocessing part of automatic optic disc detection methods is the removal of retinal blood vessels from fundus images. Retinal blood vessels are usually removed from fundus images because they intersect the boundary of the optic disc, making it more difficult to detect it. In order to compensate for this situation, many automatic optic disc segmentation approaches employ the removal of retinal blood vessels from fundus images. Examples of these ap- proaches can be found in $[11,13,14,15]$. Methods that are used for blood vessels removal from fundus images are numerous, and some examples would be morphological closing [11] and local entropy thresholding and inpainting techniques [13]. Other approaches for automatic optic disc segmentation do not remove blood vessels from fundus images, but rather use the fact that the optic disc is a point of convergence for retinal blood vessels in automatic optic disc segmentation. Examples of these approaches can be found in $[1,16]$.

When it comes to image enhancement for optic disk segmentation, various techniques can be used. For example, illumination equalization was used in [1], anisotropic diffusion was used in [17], median filter of size $15 \times 15$ pixels was used in [4], and averaging filter of size $25 \times 35$ pixels was used in [18].

Methods used for automatic optic disc detection encompass a wide range of different techniques that range from template matching (e.g. [19] (the authors used histograms instead of images for templates) and [45] (the authors used template matching for optic disc centre detection)) to algorithms inspired by biological organisms (e.g. firefly algorithm in [4] and ant colony optimization algorithm in [17]).

Methods for automatic optic disc detection that are based on deep learning are still relatively novel. Existing methods that use deep learning for automatic optic disk segmentation, localization and/or detection include $[8,21,22]$.

Approaches for automatic optic disc segmentation can be trained and evaluated on various fundus image datasets. In this paper we decided to train the proposed method on DRIVE (Digital Retinal Images for Vessel Extraction) $[23,24]$ and DIARETDB1 (Diabetic Retinopathy Database) $[25,26]$ image datasets. DRIVE consists of 40 fundus images equally split into training and testing categories. For the purposes of this paper, we used all 40 images for the testing purpose, as it is commonly done when it comes to evaluation of automatic optic disc segmentation approaches. The size of the images in the DRIVE dataset is $565 \times 584$ pixels. DIARETDB1 consists of 89 fundus images whose size is $1500 \times 1152$ pixels. Since there are no formal ground truth segmentations of the optic disc for neither of these two image datasets, we created our own optic disc manual segmentations for both of them. The proposed method was evaluated on 200 images from the MESSIDOR image dataset [44].

Our contributions in this work are:

We proposed a novel fully automatic model for detecting glaucoma disease based on deep learning approach. Model preforms semantic segmentation of optic disc and optic cup respectively.

\subsection{OPTIC CUP SEGMENTATION}

Automatic optic cup segmentation is usually associated with automated glaucoma detection and analysis. 
Image processing based glaucoma detection has been studied in the literature (e.g. [3]), and they usually work by calculating the CDR ratio. In this section of the paper we will briefly discuss some of the methods used for automatic, image processing based optic cup segmentation.

Akram et al. [3] proposed a method for optic cup segmentation that selects pixels that have intensity values that are in the top $20 \%$ from the segmented optic disc region. Lim et al. [8] proposed a method for optic cup segmentation that. In [13] authors proposed a method for optic cup segmentation that includes an over-segmentation of the optic disc region into superpixels, extraction of features belonging to these superpixels, and the classification procedure (by using the SVM classifier) that determines if the superpixel belongs to the optic cup or not.
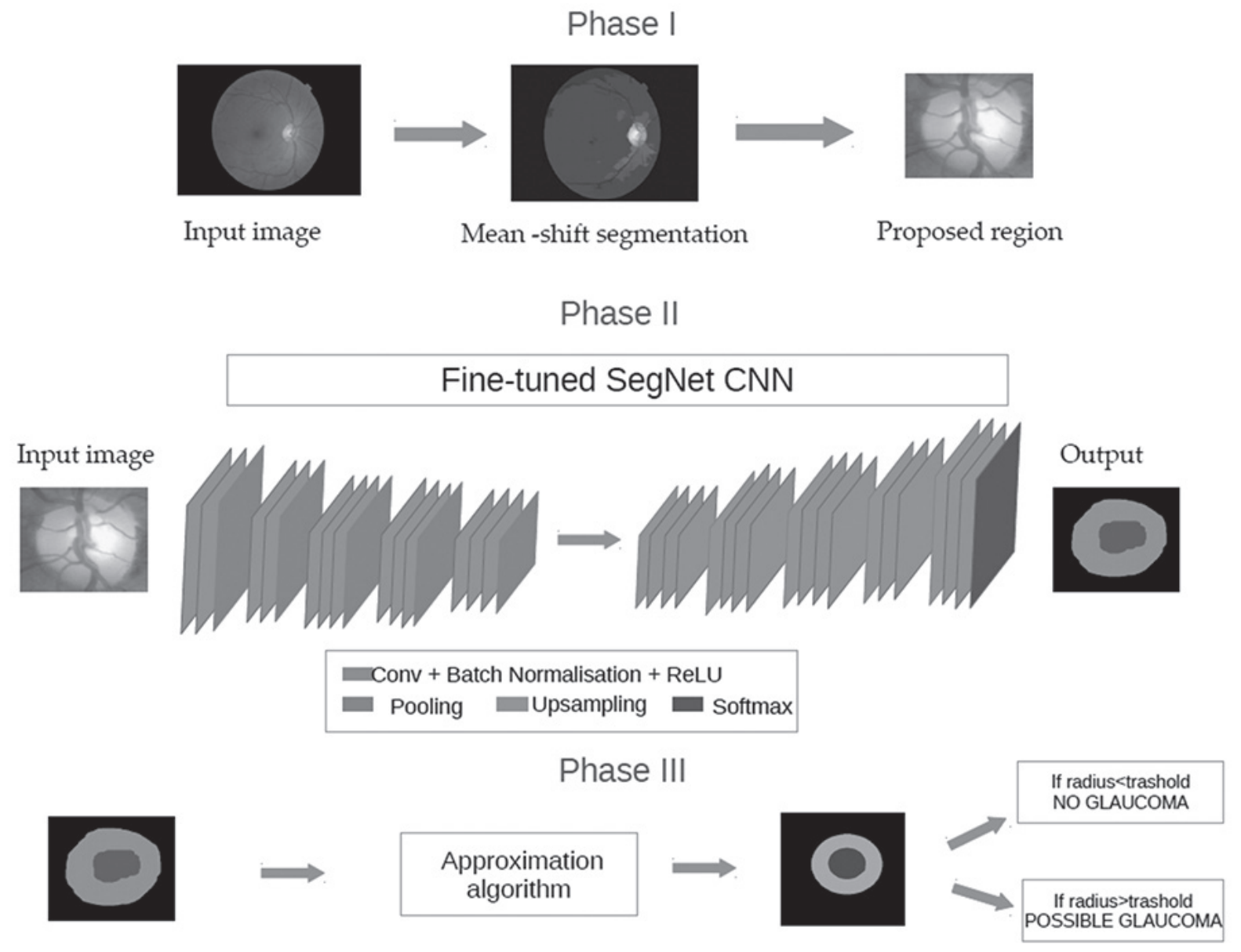

Fig. 1. Flow chart of proposed method for detecting glaucoma

\section{PROPOSED METHOD}

Fig. 1. illustrates the proposed method and is composed of three phases. Phase one, the image is

segmented using Mean-shift algorithm into set of regions. The output regions of an algorithm are taken as the locations of optic disc and respectively optic cup, with the assumption that one region may represent one of them, or both. Second phase is the process of extraction of features, where we usea window around the possible region as input to a pre-trained SegNet neural network, also called encoder - decoder network. Result is a semantic segmentation of a candidate region. Final step in our method is calculation of cup-todisc ratio (CDR) from output image of phase two. Comparing ratio with threshold value our system provide evaluation about possible glaucoma presents.

\subsection{OVER-SEGMENTATION OF THE FUNDUS IMAGES}

Segmentation is one of the most complex computer vision. There is a large range of segmentation procedures that have been developed. These procedures can be classify to the algorithms that are edge based.

Mean-shift algorithm [43] was used, because his feature-space analysis is robust and that kind of a approach can be applied in different computer vision tasks. It can be used to smoothing, mode seeking, clustering, visual tracking, discontinuity preservation and image segmentation tasks. Mean-shift use the Parzen window kernel for the estimation of density, where dense regions in feature space corresponds to local maxima or modes. So for every data point, gradient ascent will be preformed on the local estimated density until convergence. All stationary points that were ob- 
tained via gradient ascent will represent the modes of the density function. Same cluster is represented with the same stationary points. For data samples $\left\{x_{i}\right\}_{i}^{n}=1$ in the $d$-dimensional space, for sample $x$ the kernel for density estimation is given by:

$$
\hat{f}(x)=\frac{1}{n h^{d}} \sum_{i=1}^{n} K\left(\frac{x-x_{i}}{h}\right)
$$

where $K$ is kernel and $h$ is bandwidth. For feature space analysis the main step is finding the modes of it's density. The modes are usually located near the zero value of the gradient. The Mean-shift algorithm is an way for localization of zero values without using the density estimation. This is because the data is represent in features space in a spatial range. The spatial domain represents the locations for various pixels, whereas the range domain denotes the spectral signals for various spectral channels. For all this, multivariate kernel for estimation of joint density can be written as:

$$
K_{h_{s}, h_{r}}=\frac{\rho}{h_{s}^{2} h_{r}^{d}} k\left(\frac{x^{s}}{h_{s}}\right) k\left(\frac{x^{r 2}}{h_{r}}\right)
$$

where $\boldsymbol{\rho}$ represents parameter for normalization and $\boldsymbol{h}$ is used as kernel for bandwidth filtering. If we want to use Mean-shift for segmentation task bandwidth parameter is required for resolution of the detection. Mean-shift segmentation algorithm is not suitable for segmentation of resolution large images. One of the possible solutions is dividing large images into smaller patches. If we use Mean-shift segmentation in this way, there aren't any negative side effects on our result. On Fig 2. is shown example of applying Mean-shift on fundus image.

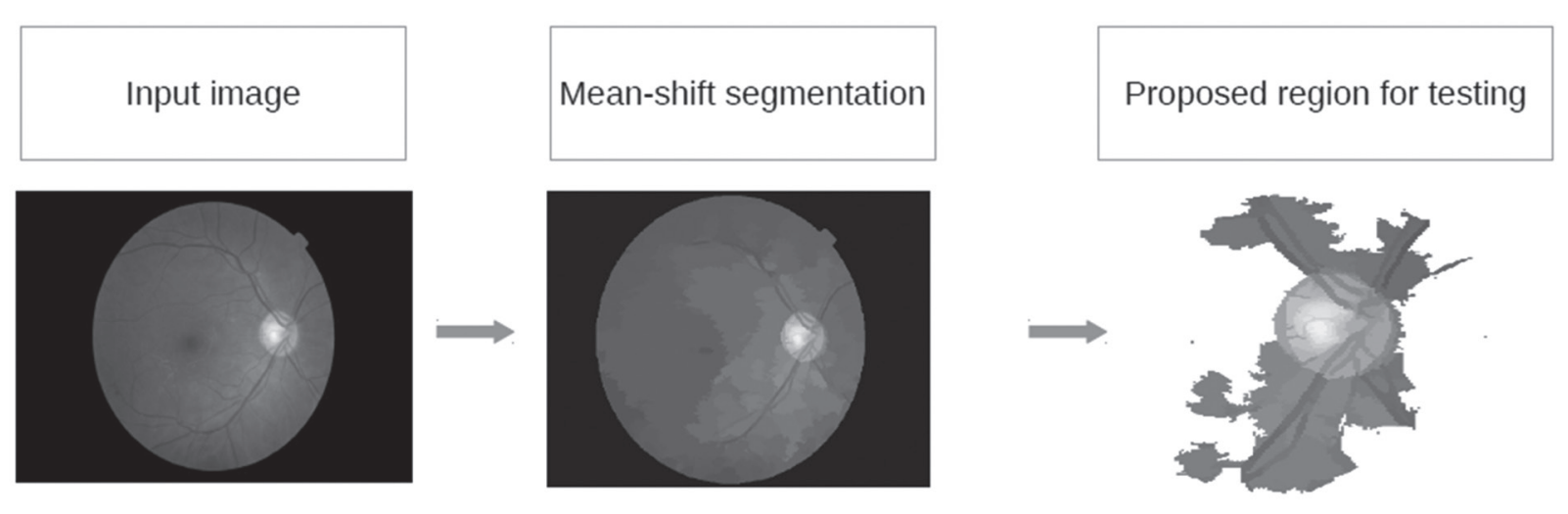

Fig. 2. On left side is original image, in the middle is Mean-shift segmentation and on right side is a region proposal.

In our method Mean-shift give us opportunity to extract region of interest. By detecting regions of interest we automatically speed up model, because large regions are removed and small region are almost surely presenting optic disc with optic cup.

\subsection{REGION CANDIDATE EXTRACTION}

Deep Convolutional Neural Networks (DCNNs) has been the best choice for document recognition since LeCun [30], but only in newly days become the stateof-art of large vision problems. During the last years DCNNs shown that they can be used to solved very complex computer vision problems problem, such as classification [27,28,29], object detection, segmentation, fine-grained categorization and among others. DCNNs are usually train in end to end mode and are shown better results than a classic feature representation (SIFT and HOG). Good thing in DCNNs is the feature invariance on image transformation. DCNNs are consist of various layers: convolutional, pooling, normalization and fully connected.

Convolutional layers are the basic block of the CNN. Every convolution layer is represented as a set of filters that are learnable. From spatial view filters are relatively small, but they goes through the all input image depth. During the process of convolution of the filters and input image the feature maps are produced. This feature maps are given as input to a function that is non-linear such as leaky rectified linear unit (LeakyReLU). Optional normalization of activation function output is done.

Pooling Layer operates independently on every depth slice of the input and resizes it spatially, using the MAX operation. The most common form is a pooling layer with filters of size $2 \times 2$ applied with a stride of 2 downsamples every depth slice in the input by 2 along both width and height, discarding 75 of the activations. Every MAX operation would in this case be taking a max over 4 numbers (little $2 \times 2$ region in some depth slice). The depth dimension remains unchanged.

Fully connected layer (FC) is the main block of DCNNs and is in charge for decision, neurons from the previous layer are taken and connected to all others. If task is classification. At the end of the network a softmax function is added and sum of neurons has to be same as the class number. At the end the weights of the network are learned using back-propagation algorithm. 
In this paper we used deep fully convolutional neural network model based on the encoder-decoder architecture, like VGG16 FCN, where the fully connected layers are removed. The decoder phase is inspired by SegNet [20] model for semantic pixel-wise segmentation. In decoder part of a network, each block has to use up sampling that needs to be non liner in order to get the complete map of features that was down sampled in max pooling layers and it's indices was produced during each pooling layer in the encoder phase of the network. SegNet has an encoder network and a decoder network. At the end of the encoder-decoder architecture there is a final pixel-wise classification layer. Each encoder in the encoder network performs convolution with a filter bank to produce a set of feature maps. The decoder network up-samples its input feature map using the max-pooling indices that was memorized in the encoder feature map. With this step we get sparse feature map.

Originally SegNet was developed for road scene semantic segmentation. In our experiment we used fine-tuned VGG16 model for encoder part of network with trained weights and adjust decoder network by creating our own classes for optic disc and optic cup. Additionally third class is background class. On Fig. 3. schema of fine-tuned network is shown.

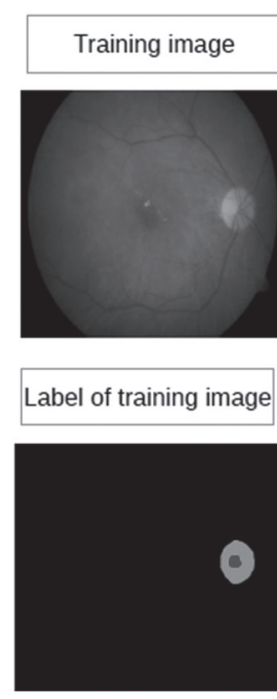

\section{Training phase}
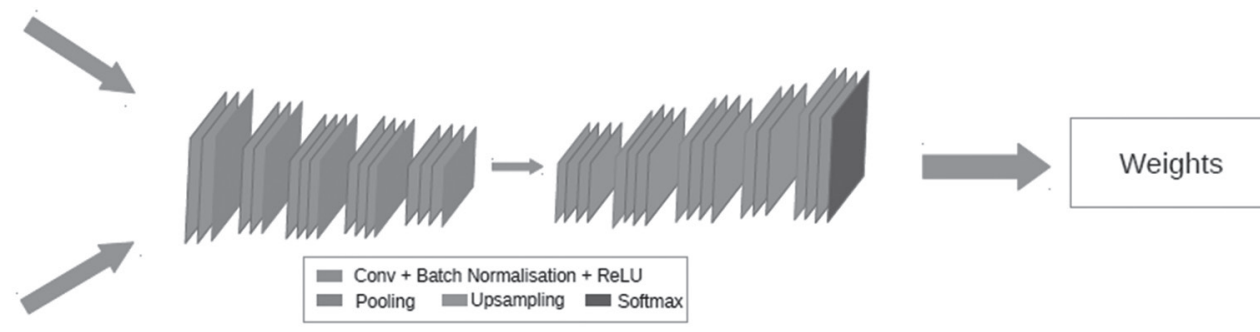

Fig. 3. Fine-tuned convolutional network schema.

\subsection{CALCULATING POSSIBLE PRESENTS OF GLAUCOMA}

Glaucoma is a disease that damages eyes optic nerve. It usually happens when fluid builds up in the front part of eye and increases the pressure in eye, damaging the optic nerve. One of possible indications that person developed this disease is calculating ratio of optic cup and optic disc. Normal cup-to-disc (CDR) ratio is 0.3 , larger CDR ratio may imply glaucoma or other pathology.

Phase III of our proposed model calculates CDR. In the existing methods, the glaucoma analysis of the eye is mainly focused on the retina. In some recent work [46], the presence of glaucoma in fundus images is predicted by classification using SVM and deep feature learning [47], there by bypassing the OD-OC segmentation.

Since the output of DCNN is semantic segmented fundus image, first step in CDR calculation was to find mass center for segmented optic cup, presented by the blue area of image. After finding mass center of blue shape we calculate 1000 radius's in different directions from mass center to the end of shape or exactly till the first pink pixel. Algorithm is repeated for pink area of the image that present optic disc. Final radius of optic cup and optic disc is calculated using confidence intervals for expectation values. Data for this kind of approach needs to fit normal distribution. Since our data (radius values) did not fit to normal distribution, yet fitted to left skewed distribution they required a reflected transformation. First we needed to reflect our data and then we applied Square-Root transformation in order to fit our data to normal distribution. For all 1000 values of radius's of optic disc and optic cup mean value and standard deviation where calculated individually. Mean value was calculated as $\bar{x}=\sum_{i=1}^{1000}\left(\frac{x_{i}}{1000}\right)$ and standard de viation was calculated as $\sigma=\sqrt{\frac{1}{1000} \sum_{i=1}^{1000}\left(x_{i}-\mu\right)^{2}}$, where $\mu$ is arithmetic mean. To find confidence interval we used $2 / 3$ rule, which specifies that the interval

$$
[\bar{x}-\operatorname{sem}(x), \bar{x}+\operatorname{sem}(x)]
$$

has probability of $2 / 3$ to contain mean value, where $\operatorname{sem}(x)$ is

$$
\operatorname{sem}(x)=\frac{s d(x)}{\sqrt{1000}}
$$

If ratio of two calculated intervals containing value over 0.3 possible glaucoma presence is detected. Using all previously described methods, our proposed algo- 
rithm for calculation of CDR is given in the following frame. Workflow of finding CDR is shown on Fig. 4.

\section{Algorithm 1 Calculating CDR}

Input: Sequence of $\mathrm{N}$ images

for all images do

find mass center on i image for blue pixel

find 1000 distances in random directions from mass center

find mass center on i image for pink pixel

find 1000 distances in random directions from mass center

calculate mean radius for two countur

\section{Output: CDR}
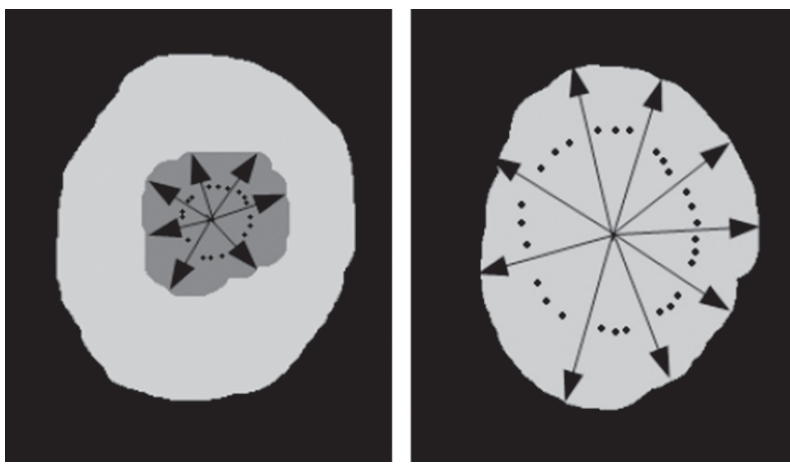

Fig. 4. Workflow of finding CDR

\section{RESULTS}

\subsection{DATASET DESCRIPTION AND EXPERIMENT SETUP}

To evaluate the effectiveness of the proposed glaucoma detection method we used fundus images from DRIVE (Digital Retinal Images for Vessel Extraction) $[23,24]$ containing 40 fundus images and DIARETDB1 (Diabetic Retinopathy Database) [25,26] containing 89 fundus images. Before dividing fundus images into separate sets, images were preprocessed. We manually segmented each image in order to prepare them for DCNN training and testing. Segmented image is composed of three components optic cup, optic disc and background. Optic cup is presented by blue pixels, optic disc with pink pixels and background is black. After preprocessing fundus images we divided data set in three components: training set, testing set and ground truth set. Ground truth dataset is label of training dataset, presenting a class that each part of training image should belong.

Step 1 includes that all test images are segmented to a regions that are homogeneous. For this task the Meanshift procedure was used. Since the regions that were extracted differ in sizes and knowing that the optic disc size is usually around 400×300 pixels, we did not inspect the regions that are bigger of the given size. So, the regions which had bounding box size that was larger than 400 were eliminated. All the remain regions which size was suitable (smaller than limit), a window in size of 400 $\times 300$ pixels was bounded around this regions and then the normalization to $480 \times 360$ pixels was done. Prepared data is send to DCNN for feature extraction. Step 2 consist of running the algorithm on every segmented image size 480 × 360 pixels through glaucoma detection algorithm described in Section 3.3.

\subsection{ASSESSMENT METHOD}

In order to assess performances of proposed model for optic disc and optic cup segmentation we used a pixel-level classification accuracy. Consequently, these measures use the pixel-level confusion matrix $C$, which aggregates predictions for the whole dataset $D$ :
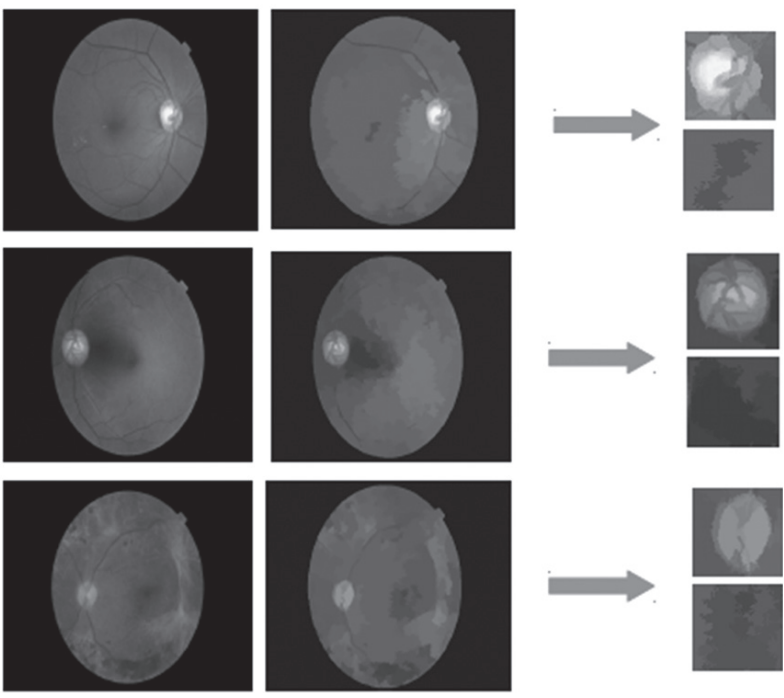

Fig. 5. Example of segmentation output.

$$
\begin{aligned}
& C_{i j}=\sum_{I \in \mathrm{D}} \mid\{z \in I \text { such that } \\
& \left.S_{g t}^{I}(z)=i \text { and } S_{p s}(z)=j\right\} \mid
\end{aligned}
$$

where $S_{g t}^{I}(z)$ is the ground-truth label of pixel $z$ in image $I, S_{p s}^{I}$ is the predicted label, and $|A|$ is s the cardinality of the set $A$. In other words, $C_{i j}$ is the number of pixels having ground-truth label $i$ and whose prediction is $j$. We denote by $G_{i}=\sum_{j=1}^{L} C_{i j}$, the total number of pixels labelled with $i$, where $L$ is the number of classes, and by $P_{j}=\sum_{j=1}^{L} C_{i j}$ the total number of pixels whose prediction is $j$. We can compute the two following measures from $C$ overall pixel accuracy measure, per- class accuracy measure. We obtain $94.5 \%$ accuracy for optic disc segmentation and $93.1 \%$ for optic cup segmentation.

For glaucoma detection, we used the measure for accuracy based on the producers and the users, that is defined as $P_{a c c}=T P / N$ and $U_{a c c}=T P /(T P+F P)$, where $T P$ are the true positives, FP are the false positives and and $N$ is the real number showing presence of glaucoma. 


\subsection{DETECTION RESULTS}

Proposed method was evaluate on fundus images from MESSIDOR database. On Fig. 6. segmentation of proposed model is present. Fig. 7. shows ratio of glaucoma present fundus images and healthy fundus images in train and test databases. In test database there are 25 fundus images with diagnosed glaucoma. In Table 1. we present results of optic disc and optic cup segmentation. Also we presented comparation of our proposed method and existing methods for glaucoma detection in Table 2. Our proposed method has success rate of $96.75 \%$, compered to other methods.

Table 1. Results of optic disc and optic cup segmentation.

\begin{tabular}{|ccc|}
\hline Method & Optic disc & Optic cup \\
\hline Superpixel [37] & 0.94 & 0.80 \\
\hline FCN [38] & 0.949 & 0.843 \\
\hline U-Net [39] & 0.962 & 0.872 \\
\hline Proposed method & $\mathbf{0 . 9 7 3}$ & $\mathbf{0 . 8 8 1}$ \\
\hline
\end{tabular}

Original image
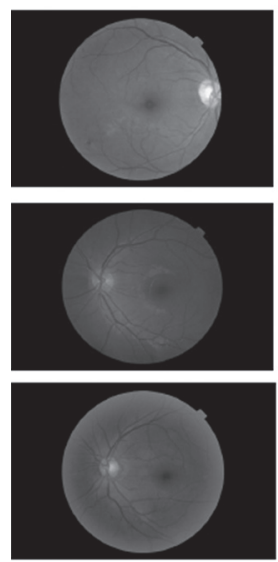

Ground truth

Our method
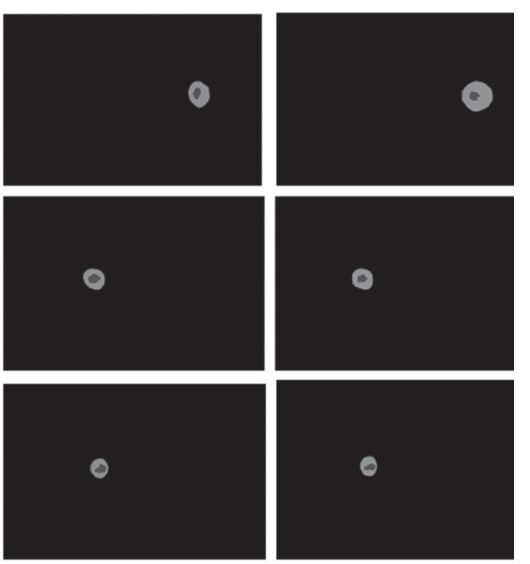

Fig. 6. Example of segmentation output.

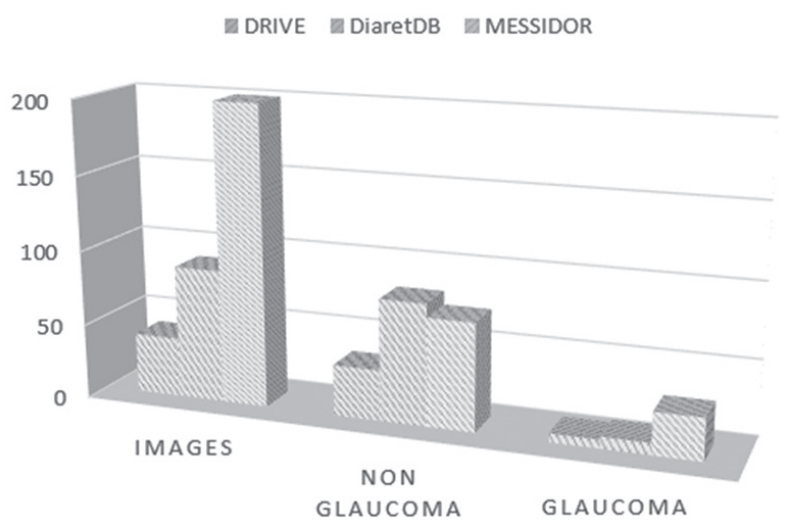

Fig. 7. Comparation of number of images in different datasets
Table 2. Results of glaucoma detection.

\begin{tabular}{|c|c|}
\hline Methods & Success rates (Acc) \\
\hline $\begin{array}{c}\text { [40] 2019, Transfer Learning. OD as ROI } \\
\text { extraction, image scaling, VGG-19 used for } \\
\text { transfer learning }\end{array}$ & $94 \%$ \\
\hline $\begin{array}{l}\text { [41] 2018, Features are extracted from CNN } \\
\text { ResNet-50 with pre-trained weights from } \\
\text { ImageNet challenge, Logistic Regression } \\
\text { Classifier, RIM-ONE r2 as dataset }\end{array}$ & $90 \%$ \\
\hline $\begin{array}{l}\text { [42] 2018, Multi-branch neural network } \\
\text { (MBNN), use of Domain Knowledge: } \\
\text { CDR,RNFLD,PPA and Patient data }\end{array}$ & $91.5 \%$ \\
\hline Proposed Method & $96.75 \%$ \\
\hline
\end{tabular}

\section{CONCLUSION}

This paper presented a novel, deep learning based approach for the automatic optic disc and optic cup segmentation. In addition the model is capable to calculate cup-to-optic ratio (CDR) and decide about potential glaucoma.

Thanks to the convolutional nature of the models and by using an efficient GPU implementation, the resulting segmentation system is very fast. The time needed to segment an entire fundus image with DCNN architecture is 1.25 seconds making our proposed model practical segmentation method. Our future work will include on expansion of our research to classification of different eye diseases using DCNN.

\section{ACKNOWLEDGEMENT}

This work was partly supported by the Ministry of Science, Education and Sport of the Republic of Croatia under grant "ViO - Vision Based Intelligent Observers" (in Croatian: "ViO - Vidom temeljeni inteligentni opserveri"). This work is part of activities of ACROSS Centre of Research Excellence for Advanced Cooperative Systems (http://across.fer.hr).

\section{REFERENCES:}

[1] Adam Hoover, Michael Goldbaum, "Locating the Optic Nerve in a Retinal Image Using the Fuzzy Convergence of the Blood Vessels", IEEE Transactions on Medical Imaging, Vol. 22, No. 8, 2003, pp. 951-958.

[2] José Pinão, Carlos Manta Oliveira, "Fovea and optic disc detection in retinal images with visible lesions", Doctoral Conference on Computing, Electrical and Industrial Systems, DoCEIS 2012: Technological Innovation for Value Creation, IFIP AICT 372, 2012, pp. 543-552.

[3] M. Usman Akram, Anam Tariq, Shehzad Khalid, M. Younus Javed, Sarmad Abbas, Ubaid Ullah Yasin, "Glaucoma detection using novel optic disc localization, hybrid feature set and classification tech- 
niques", Australasian Physical \& Engineering Sciences in Medicine, Vol. 38, No. 4, 2015, pp. 643-655.

[4] Javad Rahebi, Firat Hardalaç, "A new approach to optic disc detection in human retinal images using the Firefly algorithm", Medical \& Biological Engineering \& Computing, Vol. 54, No. 2, 2016, pp. 453-461.

[5] Kemal Akyol, Baha Sen, Safak Bayir, "Automatic detection of optic disc in retinal image by using keypoint detection, texture analysis, and visual dictionary techniques", Hindawi Publishing Corporation, Computational and Mathematical Methods in Medicine, Vol. 2016, 2016, pp. 10.

[6] K. P. Indira, J. Suganthi Vinodhini, J. Adeline Sneha, "Optic disc and optic cup segmentation for glaucoma screening adopting superpixel classification", International Journal Of Pharmacy \& Technology, Vol. 7, No. 1, 2015, pp. 8390-8400.

[7] K. Kavitha, M. Malathi, "Optic disc and optic cup segmentation for glaucoma classification", International Journal of Advanced Research in Computer Science \& Technology (IJARCST 2014), Vol. 1, No. Special 1, 2014, pp. 87-90.

[8] Vijay Badrinarayanan, Alex Kendall and Roberto Cipolla "SegNet: A Deep Convolutional EncoderDecoder Architecture for Image Segmentation." PAMI, 2017.

[9] Ahmed Wasif Reza, C. Eswaran, Subhas Hati, "Automatic tracing of optic disc and exudates from color fundus images using fixed and variable thresholds", Journal of Medical Systems, Vol. 33, 2009, pp. 73-80.

[10] Noor Elaiza Abdul Khalid, Noorhayati Mohamed Noor, Norharyati Md. Ari, “Fuzzy c-means (FCM) for optic cup and disc segmentation with morphological operation", International Conference on Robot PRIDE 2013-2014 - Medical and Rehabilitation Robotics and Instrumentation, ConfPRIDE 2013-2014, Procedia Computer Science, Vol. 42, 2014, pp. 255-262.

[11] Fatma A. Hashim, Nancy M. Salem, Ahmed F. Seddik, "Automatic segmentation of optic disc from color fundus images", Jökull Journal, Vol. 63, No. 10, October 2013, pp. 142-153.
[12] Thomas Walter, Jean-Claude Klein, "Segmentation of color fundus images of the human retina: Detection of the optic disc and the vascular tree using morphological techniques", International Symposium on Medical Data Analysis, ISMDA 2001: Medical Data Analysis, LNCS 2199, 2001, pp. 282-287.

[13] G. Arumugam, S. Nivedha, “Optic disc segmentation based on independent component analysis and kmeans clustering", International Journal of Emerging Trends \& Technology in Computer Science (IJETTCS), Vol. 2, No. 6, November-December 2013.

[14] Ana Salazar-Gonzalez, Djibril Kaba, Yongmin Li, Xiaohui Liu. "Segmentation of the blood vessels and optic disk in retinal images". IEEE Journal of Biomedical and Health Informatics, Vol. 18, No. 6, November 2014. pp. 1874-1886.

[15] Gopalakrishnan, A. Almazroa, K. Raahemifar, V. Lakshminarayanan, "Optic disc segmentation using circular Hough transform and curve fitting", 2nd International Conference on Opto-Electronics and Applied Optics (IEM OPTRONIX), 2015.

[16] Ana G. Salazar-Gonzalez, Yongmin Li, Xiaohui Liu, "Optic disc segmentation by incorporating blood vessel compensation", IEEE Third International Workshop On Computational Intelligence In Medical Imaging (CIMI), 2011.

[17] Carla Pereira, Luís Gonçalves, Manuel Ferreira, "Optic disc detection in color fundus images using ant colony optimization", Medical \& Biological Engineering \& Computing, Vol. 51, No. 3, March 2013, pp. 295-303.

[18] Ahmed Wasif Reza, C. Eswaran, Kaharudin Dimyati, "Diagnosis of diabetic retinopathy: automatic extraction of optic disc and exudates from retinal images using marker-controlled Watershed transformation", Journal of Medical Systems, Vol. 35, No. 6, December 2011,pp. 1491-1501.

[19] Amin Dehghani, Hamid Abrishami Moghaddam, Mohammad-Shahram Moin, "Optic disc localization in retinal images using histogram matching", EURASIP Journal on Image and Video Processing, Vol. 2012, No. 1, December 2012.

[20] Gilbert Lim, Yuan Cheng, Wynne Hsu, Mong Li Lee, "Integrated optic disc and cup segmentation with 
deep learning", IEEE 27th International Conference on Tools with Artificial Intelligence (ICTAI), 2015, pp. 162-169.

[21] Hanan S. Alghamdi, Hongying Lilian Tang, Saad A. Waheeb, Tunde Peto, "Automatic optic disc abnormality detection in fundus images: a deep learning approach", Third International Workshop on Ophthalmic Medical Image Analysis (OMIA3, MICCAI 2016), 2016.

[22] Kevis-Kokitsi Maninis, Jordi Pont-Tuset, Pablo Arbeláez, Luc Van Gool, International Conference on Medical Image Computing and Computer-Assisted Intervention, MICCAI 2016: Medical Image Computing and Computer-Assisted Intervention MICCAI 2016, pp. 140-148, Lecture Notes in Computer Science (LNCS, volume 9901), 2016.

[23] Joes Staal, Michael D. Abràmo_, Meindert Niemeijer, Max A. Viergever, Bram van Ginneken. "Ridgebased vessel segmentation in color images of the retina". IEEE Transactions on Medical Imaging, Vol. 23, No. 4, April 2004, pp. 501-509.

[24] M. Niemeijer, J. Staal, B. van Ginneken, M. Loog, M.D. Abràmo, "Comparative study of retinal vessel segmentation methods on a new publicly available database", Proceedings of SPIE - The International Society for Optic Engineering 5370, May 2004.

[25] Tomi Kauppi, Valentina Kalesnykiene, Joni-Kristian Kamarainen, Lasse Lensu, liris Sorri, Asta Raninen, Raija Voutilainen, Juhani Pietilä, Heikki Kälviäinen, Hannu Uusitalo, DIARETDB1 - Standard Diabetic Retinopathy Database, http://www.it.lut._project/imageret/diaretdb1/\#USAGE, last accessed on February $2^{\text {nd }}, 2017$.

[26] Tomi Kauppi, Valentina Kalesnykiene, Joni-Kristian Kamarainen, Lasse Lensu, liris Sorri, Asta Raninen, Raija Voutilainen, Hannu Uusitalo, Heikki Kälviäinen, Juhani Pietilä, DIARETDB1 diabetic retinopathy database and evaluation protocol, Technical report.

[27] Christian Szegedy, Wei Liu, Yangqing Jia, Pierre Sermanet, Scott Reed, Dragomir Anguelov, Dumitru Erhan, Vincent Vanhoucke, Andrew Rabinovich, "Going deeper with convolutions", IEEE Conference on Computer Vision and Pattern Recognition (CVPR), 2015.
[28] Alex Krizhevsky, Ilya Sutskever, and Geo_ Hinton. "Imagenet classification with deep convolutional neural networks", In Advances in Neural Information Processing Systems 25, 2012.

[29] Szegedy, Christian and Toshev, Alexander and Erhan, Dumitru. "Deep Neural Networks for Object Detection", Advances in Neural Information Processing Systems 26, 2553-2561, 2013.

[30] LeCun, Y., Boser, B., Denker, J. S., Henderson, D., Howard, R. E., Hubbard, W., and Jackel, L. D. "Backpropagation applied to handwritten zip code recognition". Neural Comput., Vol. 1, No.4, 1989, pp, 541-551.

[31] Wang R.“Edge Detection Using Convolutional Neural Network" In: Cheng L., Liu Q., Ronzhin A. (eds) Advances in Neural Networks - ISNN 2016. ISNN 2016. Lecture Notes in Computer Science, vol 9719. Springer, 2016.

[32] Huang, F.J.; Boureau, Y.L.; LeCun, Y. Unsupervised learning of invariant feature hierarchies with applications to object recognition. In Proceedings of the 2007 IEEE Conference on Computer Vision and Pattern Recognition, Minneapolis, MN, USA, Vol 18., June 2007; pp. 1-.8.

[33] J.Capriolli, J.M. Miller. "Optic Disc Rim Area is Related to Disc Size in Normal Subjects" 1987.

[34] Cheng, Y. "Mean shift, mode seeking, and clustering. "IEEE Trans. Pattern Anal. Mach. Intell. Vol.17, 1995, pp. 790-799.

[35] Comaniciu, D.; Meer, P, "Mean shift: A robust approach toward feature space analysis." IEEE Trans. Pattern Anal. Mach. Intell., Vol. 24, 2002, pp. 603-619.

[36] Boykov, Y.; Funka-Lea, G. Graph Cuts and Efficient N-D Image Segmentation. Int. J. Comput. Vis. , Vol. 70, 2006, pp. 109-131.

[37] Cheng J, Liu J, Xu Y, Yin F, Wong D. W. K, Tan N. M, Tao D, Cheng C. Y, Aung T, Wong T. Y (2013) „Superpixel classification based optic disc and optic cup segmentation for glaucoma screening" IEEE TransMedlmaging. https://doi.org/10.1109/ TMI.2013.2247770

[38] Long J, Shelhamer E, Darrell T (2015) „Fully convolutional networks for semantic segmentation.“ In: Proceedings of the IEEE Computer Society Conference on Computer Vision and Pattern Recognition. 
[39] Ronneberger O, Fischer P, Brox T (2015) „U-net: Convolutional networks for biomedical image segmentation." In: Lecture notes in computer science (including subseries lecture notes in artificial intelligence and lecture notes in bioinformatics).

[40] Juan J. Gómez-Valverde et al., "Automatic glaucoma classification using color fundus images based on convolutional neural networks and transfer learning", Biomedical Optics Express, Vol. 10, No. 2, 1 Feb 2019.

[41] Annan Li , Yunhong Wang , Jun Cheng , Jiang Liu, "Combining Multiple Deep Features for Glaucoma Classification", 2018 IEEE International Conference on Acoustics, Speech and Signal Processing (ICASSP).

[42] . Yidong Chai, Hongyan Liu, JieXu, "Glaucoma diagnosis based on both hidden features and domain knowledge through deep learning models", Knowledge-Based Systems, ScienceDirect, Vol. 161, No. 1, December 2018, pp.147-156.

[43] Comaniciu, D. and Meer, P., ,Mean Shift: A Robust Approach Toward Feature Space Analysis" IEEE
Transactions on Pattern Analysis and Machine Intelligence, Vol. 24, No. 5, 2002, pp. 603-619.

[44] Decencière et al..„Feedback on a publicly distributed database: the Messidor database", Image Analysis \& Stereology, Vol. 33, No. 3, 2004 p. 231-234.

[45] Chuang Wang, Djibril Kaba, Yongmin Li, Level set segmentation of optic discs from retinal images, Journal of Medical and Bioengineering, Vol. 4, No. 3, June 2015.

[46] Dey, A., \& K. Bandyopadhyay, S. (2015). Automated Glaucoma Detection Using Support Vector Machine Classification Method. Journal of Advances in Medicine and Medical Research, Vol. 11, No.12, 2015, pp. 1-12.

[47] Gulshan V, Peng L, Coram M, et al. Development and Validation of a Deep Learning Algorithm for Detection of Diabetic Retinopathy in Retinal Fundus Photographs. JAMA. Vol. 316,No. 22, 2016, pp.2402-2410. 